B. Duchemann (Avicenne), J. Dumoulin (Boulogne-Billancourt), C. Duval (Juvisy-sur-Orge), L.I. Escaut (Paris-Kremlin Bicêtre), H. Ferrand (Paris-Bichat), R. Flicoteaux (Paris-Bichat), P. Fraisse (Strasbourg), S. Gallien (Paris-Saint-Louis), S. Girard (Le Mans), C. Godet (Poitiers), M. Gousseff (Avicenne), D. Herman (Nevers), S. Jaureguiberry (Paris-Pitié-Salpêtrière), V. Joly (Paris-Bichat), J. Le Grusse (Toulouse), N. Lerolle (Paris-Saint-Louis), E. Leroy-Terquem (Meulan), D. Liné (Soissons), A. Lopes (Paris-Lariboisière), J. Macey (Paris-Cochin), G. Mellon (Paris-Bichat), J.L. Meynard (Paris-Saint-Antoine), J.C. Mouries (Bastia), J.M. Naccache (Paris-Tenon), E. Ngwem (Créteil), G. Oliviero (Longjumeau), B. Philippe (Pontoise), C. Richaud (Paris-Necker), C. Rioux (Paris-Bichat), Z. Saakashvili (Créteil), T.A. Szwebel (Paris-Hôtel Dieu) and P. Vaillant (Nancy).

The authors thank all physicians and microbiologists who provided data for the study.

\title{
References
}

1 Pontali E, Sotgiu G, D'Ambrosio L, et al. Bedaquiline and multidrug-resistant tuberculosis: a systematic and critical analysis of the evidence. Eur Respir J 2016; 47: 394-402.

2 Diacon AH, Pym A, Grobusch MP, et al. Multidrug-resistant tuberculosis and culture conversion with bedaquiline. N Engl J Med 2014; 371: 723-732.

3 Tiberi S, De Lorenzo S, Centis R, et al. Bedaquiline in MDR/XDR-TB cases: first experience on compassionate use. Eur Respir J 2014; 43: 289-292.

4 van Halsema C, Humphreys S, Bonington A. Extensively drug-resistant tuberculosis: early access to bedaquiline for a UK patient. Eur Respir J 2014; 43: 292-294.

5 Ndjeka N, Conradie F, Schnippel K, et al. Treatment of drug-resistant tuberculosis with bedaquiline in a high HIV prevalence setting: an interim cohort analysis. Int J Tuberc Lung Dis 2015; 19: 979-985.

6 Diacon AH, Dawson R, von Groote-Bidlingmaier F, et al. 14-day bactericidal activity of PA-824, bedaquiline, pyrazinamide, and moxifloxacin combinations: a randomised trial. Lancet 2012; 380: 986-993.

7 Falzon D, Gandhi N, Migliori GB, et al. Resistance to fluoroquinolones and second-line injectable drugs: impact on multidrug-resistant TB outcomes. Eur Respir J 2013; 42: 156-168.

8 Guglielmetti L, Le Dû D, Jachym M, et al. Compassionate use of bedaquiline for the treatment of multidrug-resistant and extensively drug-resistant tuberculosis: interim analysis of a French cohort. Clin Infect Dis 2015; 60: 188-194.

9 World Health Organization. The Use of Bedaquiline in the Treatment of Multidrug-resistant Tuberculosis: Interim Policy Guidance. WHO/HTM/TB/2013.6. Geneva, World Health Organization, 2013.

10 Canetti G, Rist N, Grosset J. Mesure de la sensibilité du bacille tuberculeux aux drogues antibacillaires par la méthode des proportions [Measurement of sensitivity of the tuberculous bacillus to antibacillary drugs by the method of proportions. Methodology, resistance criteria, results and interpretation]. Rev Tuberc Pneumol 1963; 27: 217-272.

11 Kurbatova EV, Cegielski JP, Lienhardt C, et al. Sputum culture conversion as a prognostic marker for end-of-treatment outcome in patients with multidrug-resistant tuberculosis: a secondary analysis of data from two observational cohort studies. Lancet Respir Med 2015; 3: 201-209.

12 Rustomjee R, Diacon AH, Allen J, et al. Early bactericidal activity and pharmacokinetics of the diarylquinoline TMC207 in treatment of pulmonary tuberculosis. Antimicrob Agents Chemother 2008; 52: 2831-2835.

13 Migliori GB, Sotgiu G, Gandhi NR, et al. Drug resistance beyond extensively drug-resistant tuberculosis: individual patient data meta-analysis. Eur Respir J 2013; 42: 169-179.

14 Balabanova Y, Ignatyeva O, Fiebig L, et al. Survival of patients with multidrug-resistant TB in Eastern Europe: what makes a difference? Thorax 2016; in press [DOI: 10.1136/thoraxjnl-2015-207638].

15 Bernard C, Veziris N, Brossier F, et al. Molecular diagnosis of fluoroquinolone resistance in Mycobacterium tuberculosis. Antimicrob Agents Chemother 2015; 59: 1519-1524.

\section{Respiratory decline is integral to disease progression in Huntington's disease}

To the Editor:

Huntington's disease is an autosomal inherited monogenetic condition in which the mutation is an expansion of the cytosine-adenine-guanine (CAG) repeat sequence at the $\mathrm{N}$-terminal end of the huntingtin gene [1]. More than 40 repeats are associated with neuronal dysfunction and death, predominantly within the striatum resulting in a triad of movement, behaviour and cognitive impairment; other symptoms include weight loss, sleep disturbance and respiratory dysfunction, which may or may not be of primary neurological origin [1-3]. Death occurs 15-30 years after onset of symptoms [1], usually due to pneumonia [4], yet it is not known whether respiratory dysfunction is a feature of late stage disease or whether it appears earlier in the disease evolution. Previous research suggests that dysregulation within the respiratory centre results in irregular breathing patterns $[5,6]$; decreased respiratory muscle strength and lung volumes have also been identified [7] which, alongside swallow dysfunction [4], could precipitate respiratory failure. Huntington's disease is a complex long-term condition and contributing factors such as swallow dysfunction, posture, 
physical inactivity and reduced exercise capacity have not yet been investigated in relation to respiratory function. We conducted a cross-sectional study aiming to characterise respiratory function across all stages of disease and explore primary and secondary contributors to respiratory decline. Given one previous report of respiratory weakness in Huntington's disease, we performed a follow-on study to assess the feasibility of home-based inspiratory muscle training in Huntington's disease.

67 participants testing positive for the Huntington's disease gene were recruited to the cross-sectional study from the South Wales Huntington's disease clinic (Cardiff, UK) between July 2009 and December 2012. Inclusion criteria were: CAG repeat $\geqslant 40$; aged $\geqslant 18$ years; able to understand instructions in English; and maintenance of a stable medical regime for 4 weeks prior to the study. Healthy controls $(n=39$; matched for age, sex, smoking pack-years and fat-free mass) were recruited from relatives, carers and members of Cardiff University. Informed consent was obtained under the Research Ethics Committee for Wales (08/MRE09/65)

Huntington's disease-positive participants were categorised as pre-manifest or manifest based on the presence of motor abnormalities representing an unequivocal diagnosis of Huntington's disease; disease severity was categorised by the Unified Huntington's Disease Rating Scale (total functional capacity scale ranging 0-13) [8]. Outcome measures were: forced vital capacity; forced expiratory volume in $1 \mathrm{~s}$ (FEV1)/peak expiratory flow rate (PEFR) ratio; peak cough flow (PCF); maximal inspiratory pressure (MIP); sniff nasal inspiratory pressure (SNIP), maximal expiratory pressure; swallow capacity measured as $\mathrm{mL} \cdot \mathrm{s}^{1}{ }^{1}$ during timed swallow of $150 \mathrm{~mL}$ of water [9]; metabolic equivalents via the international physical activity questionnaire (short form) [10]; 6-min walk distance (20-m circuit); and thoracic posture via bespoke digital analysis [11].

Statistically significant differences were found for all respiratory function variables across healthy, pre-manifest and manifest participants (table 1). Post hoc analyses identified decreased respiratory function in the manifest group compared to healthy controls and pre-manifest groups, with no differences between healthy controls and pre-manifest. Analysis of FEV1/PEFR showed that eight (40\%) pre-manifest and $26(55.3 \%)$ manifest participants had a ratio of $>8$, indicative of central or upper airway obstruction [12]. In manifest participants with Huntington's disease, all measures of respiratory function significantly correlated with disease progression (Spearman's $\mathrm{r}=0.716-0.863, \mathrm{p}<0.001$ ) (figure 1). Regression analysis indicated PCF $<270 \mathrm{~L} \cdot \mathrm{min}^{-1}$ [13] when total functional capacity was 5, i.e. middle stage of the disease, suggesting that respiratory intervention may be warranted earlier in the disease process than typically considered.

Percentage predicted swallow capacity, thoracic angle, physical activity and exercise capacity significantly correlated with respiratory function in manifest participants (Spearman's $r=0.465-0.790, p<0.001$ ). Swallow capacity was normal in all pre-manifest participants and abnormal in 39 (84.8\%) manifest participants with Huntington's disease. Physical activity scores were categorised as moderate (median=1502.50 metabolic equivalent minutes.week ${ }^{-1}$; interquartile range (IQR) 2418.4) for people with pre-manifest Huntington's disease and low (82.50 metabolic equivalent minutes. $w^{-1} \mathrm{k}^{-1}$; IQR 618.80) for people with manifest disease. 6-min walk distance was $78.63 \%$ predicted and $27.73 \%$ predicted for pre-manifest and manifest participants with Huntington's disease, respectively.

TABLE 1 Comparison of healthy controls, pre-manifest and manifest participants

Healthy controls Pre-manifest Manifest

\begin{tabular}{|c|c|c|c|}
\hline Subjects & 39 & 20 & 47 \\
\hline FVC L & $3.59 \pm 0.92(3.29-3.88)$ & $3.93 \pm 0.97(3.48-4.38)$ & $2.14 \pm 1.39^{\#, 9}(1.74-2.55)$ \\
\hline FVC \% pred & $94.00 \pm 15.89$ & $101.15 \pm 13.84$ & $56.70 \pm 31.69^{\#, 9}$ \\
\hline FEV 1 L & $3.02 \pm 0.83(2.75-3.29)$ & $3.31 \pm 0.79(2.94-3.68)$ & $1.88 \pm 1.20^{\# .9}(1.53-2.23)$ \\
\hline PEFR L-min ${ }^{-1}$ & $457.41 \pm 113.22(420.71-494.11)$ & $435.05 \pm 139.73(369.66-500.44)$ & $221.49 \pm 141.61^{\# .9}(179.91-263.07)$ \\
\hline PEFR \% pred & $100.13 \pm 18.71$ & $92.60 \pm 24.63$ & $49.49 \pm 29.53^{\#, 9}$ \\
\hline PCF L. $\min ^{-1}$ & $433.95 \pm 102.48(400.73-467.17)$ & $504.55 \pm 124.12(446.46-562.64)$ & $269.46 \pm 154.5^{\# .9}(220.67-318.25)$ \\
\hline SNIP $\mathrm{cmH}_{2} \mathrm{O}$ & $74.13 \pm 31.68(63.72-84.54)$ & $81.32 \pm 33.22(65.31-97.33)$ & $34.42 \pm 23.63^{\# .9}(27.15-41.69)$ \\
\hline SNIP \% pred & $77.97 \pm 32.84$ & $85.29 \pm 32.69$ & $36.65 \pm 26.62^{\#, \pi}$ \\
\hline
\end{tabular}

Data are presented as mean \pm SD with $95 \%$ confidence intervals. FVC: forced vital capacity; FEV1: forced expiratory volume in 1 s; PEFR: peak expiratory flow rate; PCF: peak cough flow; MIP: maximal inspiratory pressure; SNIP: sniff nasal inspiratory pressure. ${ }^{\#}$ : difference between

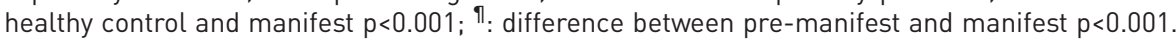



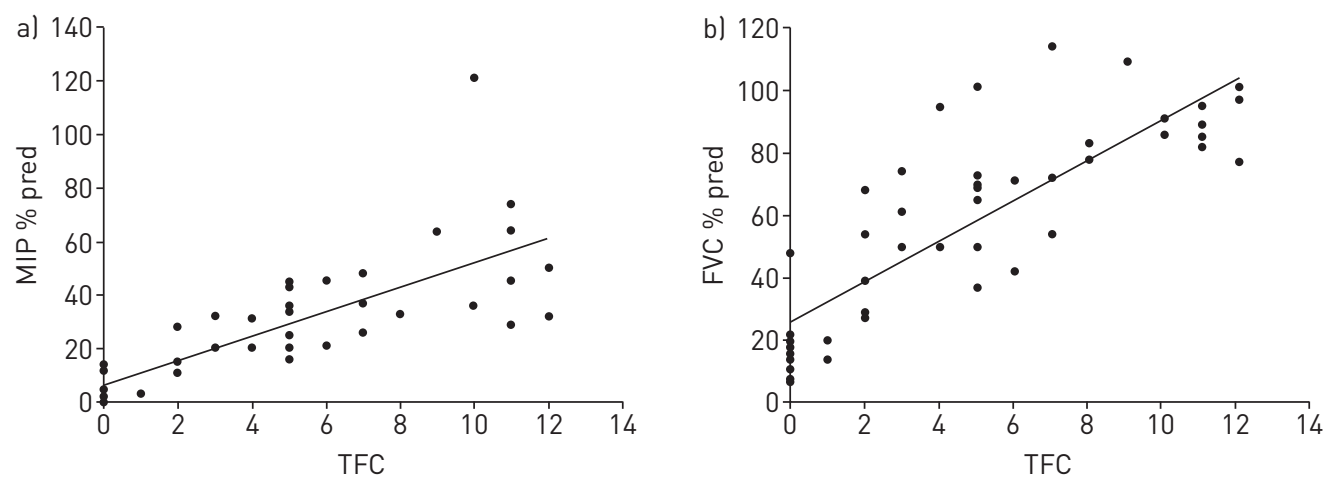

FIGURE 1 Respiratory function and disease progression in people with manifest Huntington's disease. a) Respiratory muscle strength and total functional capacity (TFC). b) Lung volume and TFC. MIP: maximal inspiratory pressure; FVC: forced vital capacity.

Participants for the follow-on training pilot were selected from the cross-sectional cohort (Ethics: 11/WA/ 0183; and ISCRTN90741776). Inclusion and exclusion criteria were as for the cross-sectional study, additionally participants were excluded if they were pre-symptomatic and had an MIP $>80 \%$ pred. 20 participants were randomly allocated to intervention or placebo using a minimisation method [14] to match for age, sex and smoking habit and were blinded to group allocation. The intervention group ( $\mathrm{n}=10)$ used the POWERbreathe ${ }^{\circledast} \mathrm{K} 3$ device (POWERbreathe International Ltd, Southam, UK) for 30 breaths, twice a day at a resistance of $50 \%$ of MIP for 6 weeks; resistance was set at $10 \mathrm{cmH}_{2} \mathrm{O}$ for the placebo group. The POWERbreathe ${ }^{\circ} \mathrm{K}$ device automatically sets resistance based on MIP pressures generated in the first two inspirations. Although resistance based on SNIP would have been more appropriate for the sample, this device provided resistance throughout the range of movement of the inspiratory muscles. A habituation period of 1 week preceded the study. Participants were instructed by the researcher who provided support through alternate weekly phone calls and home visits. The primary outcome measure was SNIP and the secondary outcome was cough efficacy as measured by PCF, the measurement was not blinded. Adherence was measured as number of training sessions as recorded by the POWERbreathe ${ }^{\oplus} \mathrm{K}$ device.

All participants had MIP and SNIP $<80 \%$ pred. Adherence to inspiratory muscle training was similar for both intervention and placebo groups: $70.67 \pm 26.35 \%$ and $74.53 \pm 21.03 \%$, respectively. For those that completed the study, SNIP increased from $47.60 \pm 33.96 \mathrm{cmH}_{2} \mathrm{O}$ to $53.60 \pm 28.31 \mathrm{cmH}_{2} \mathrm{O}$ (intervention: $\mathrm{n}=5$ ) and from $46.00 \pm 20.06 \mathrm{cmH}_{2} \mathrm{O}$ to $53.00 \pm 18.17 \quad \mathrm{cmH}_{2} \mathrm{O}$ (placebo: $\mathrm{n}=7$ ) and PCF increased from $415.80 \pm 153.57 \mathrm{~L} \cdot \mathrm{min}^{-1}$ to $448 \pm 144.97 \mathrm{~L} \cdot \mathrm{min}^{-1}$ (intervention) and from $334.00 \pm 101.64 \mathrm{~L} \cdot \mathrm{min}^{-1}$ to $370.71 \pm 89.00 \mathrm{~L} \cdot \mathrm{min}^{-1}$ (placebo). With nonsignificant increases in SNIP and PCF within both groups, further analyses were conducted pooling the groups to explore whether participating in the programme, irrespective of resistance, changed respiratory function. Post-intervention mean difference for SNIP was $8.07 \mathrm{cmH}_{2} \mathrm{O}$ (effect size 0.36) and PCF $49.39 \mathrm{~L} \cdot \mathrm{min}^{-1}$ (effect size 0.37). The small effect sizes suggest that regular breathing exercises, irrespective of added resistance, could improve cough efficacy and respiratory muscle strength.

We present data that gives a clear indication that respiratory failure in Huntington's disease is not simply a consequence of a neurodegenerative condition. The decline in respiratory function is integral to the disease process and we suggest that regular breathing exercises may increase the capacity of the respiratory muscles and improve cough effectiveness. This study expands on previous research findings of reduced lung volume and respiratory muscle strength in Huntington's disease [7] by identifying that: upper airway changes occur in pre-manifest Huntington's disease; cough effectiveness is reduced in the mid stage of the disease; and posture, physical activity and exercise capacity influence respiratory function in Huntington's disease. Our data provides a holistic representation of respiratory function in Huntington's disease across the disease life cycle. We present comprehensive data of physiological variables of decreased lung volume and respiratory muscle strength and upper airway obstruction that are responsible for respiratory failure in people with Huntington's disease and suggest that clinicians monitor respiratory function from at least the middle stage of the disease. Further research is needed to identify the appropriate interventional strategies based on confirmation of feasibility and effectiveness of regular breathing exercises in this study. respiratory failure http://ow.ly/YXTt300mIQw 
Una Jones $\oplus^{1}$, Monica Busse ${ }^{1}$, Stephanie Enright ${ }^{1}$ and Anne E. Rosser ${ }^{2,3}$

${ }^{1}$ School of Healthcare Sciences, Cardiff University, Cardiff, UK. ${ }^{2}$ Neurosciences and Mental Health Institute, Cardiff

University, Cardiff, UK. ${ }^{3}$ Cardiff Brain Repair Group, School of Biosciences, Cardiff University, Cardiff, UK.

Correspondence: Una Jones, School of Healthcare Sciences, Cardiff University, Heath Park, Cardiff, CF14 4XN, UK.

E-mail: jonesuf@cardiff.ac.uk

Received: Dec 132015 | Accepted after revision: April 282016 | First published online: June 232016

Clinical trials: This study is registered at ISRCTN with the identifiers ISRCTN90741776 and ISRCTN72770961

Support statement: Funding was received from the European Huntington's Disease Network, Physiotherapy Research Foundation UK and Research Capacity Building Collaboration Wales. Funding information has been deposited with Open Funder Registry.

Conflict of interest: Disclosures can be found alongside the online version of this article at erj.ersjournals.com

\section{References}

1 Bates GP. The molecular genetics of Huntington disease - a history. Nat Rev Genet 2005; 6: 766-773.

2 Ross CA, Tabrizi SJ. Huntington's disease: from molecular pathogenesis to clinical treatment. Lancet Neurol 2011; 10: 83-98.

3 Bano D, Zanetti F, Mende Y, et al. Neurodegenerative processes in Huntington's disease. Cell Death Dis 2011; 2 : e228.

4 Heemskerk AW, Roos RAC. Aspiration pneumonia and death in Huntington's disease. PLoS Curr 2012; 4: RRN1293.

5 Fischer J, Oepen G, Matthys H. Typical breathing pattern in patients with Huntington's chorea. Biol Psychol 1983; 16: 286.

6 Leopold NA, Kagel MC, Berkowitz L, et al. Respiratory abnormalities in Huntington's disease. J Neurol 1985; $232: 87$.

7 Reyes A, Cruickshank T, Ziman M, et al. Pulmonary function in patients with Huntington's Disease. BMC Pulm Med 2014; 14: 89 .

8 Shoulson I, Fahn S. Huntington disease: clinical care and evaluation. Neurology 1979; 29: 1-3.

9 Hughes TA, Wiles CM. Clinical measurement of swallowing in health and in neurogenic dysphagia. QJM 1996; 89: 109-116.

10 IPAQ Research Committee. Guidelines for data processing and analysis of the International Activity Questionnaire (IPAQ). 2005.

11 Jones U, Sparkes V, Busse M, et al. Reliability of digital analysis of thoracic, neck angle and head tilt measurements. J Bone Joint Surg 2011; 93: Suppl. iv, 490.

12 Pellegrino R, Viegi G, Brusasco V, et al. Interpretative strategies for lung function tests. Eur Respir J 2005; 26 : 948-968.

13 Bott J, Blumenthal S, Buxton M, et al. Guidelines for the physiotherapy management of the adult, medical, spontaneously breathing patient. Thorax 2009; 64: Suppl. 1, i1-i52.

14 Altman D, Bland J. Treatment allocation by minimisation. Br Med J 2005; 330: 843.

Eur Respir J 2016; 48: 585-588 | DOI: 10.1183/13993003.02215-2015 | Copyright @eERS 2016

ERJ Open articles are open access and distributed under the terms of the Creative Commons Attribution Non-Commercial Licence 4.0. 